\title{
XMM-Newton observations of PSR B1957+20
}

\author{
H. H. Huang and W. Becker
}

\author{
Max-Planck-Institut für extraterrestrische Physik, Giessenbachstrasse, 85748 Garching, Germany \\ e-mail: rhuang@mpe.mpg.de
}

Received 13 October 2006 / Accepted 9 January 2007

ABSTRACT

\begin{abstract}
We report on XMM-Newton observations of the "Black Widow pulsar", PSR B1957+20. The pulsar's X-ray emission is non-thermal and best modeled with a single powerlaw spectrum of photon index $2.03_{-036}^{+0.51}$. No coherent X-ray pulsations at the pulsar's spin-period could be detected, though a strong binary-phase dependence of the X-ray flux is observed for the first time. The data suggest that the majority of the pulsar's X-radiation is emitted from a small part of the binary orbit only. We identified this part as being near to where the radio eclipse takes place. This could mean that the X-rays from PSR B1957+20 are mostly due to intra-shock emission which is strongest when the pulsar wind interacts with the ablated material from the companion star.
\end{abstract}

Key words. pulsars: individual: PSR B1957+20 - stars: neutron - X-rays: stars - binaries: eclipsing

\section{Introduction}

Until now, more than 1700 rotation-powered radio pulsars are detected. Among them are about $10 \%$ which are millisecond pulsars (MSPs) (Manchester et al. 2005). They form a separate population. The majority of them resides in Globular Clusters (cf. Bogdanov et al. 2006). MSPs are presumed to have been spun up in a past accretion phase by mass and angular transfer from a binary companion (Alpar et al. 1982). Only about one third of them are seen to be solitary. It is believed that they lost their companion, e.g. in a violent supernova event. All MSPs possess very short spin periods of less than $20 \mathrm{~ms}$ and show a high spin stability with period derivatives in the range $\approx 10^{-18}-10^{-21}$. MSPs are generally very old neutron stars with spin-down ages $\tau=P / 2 \dot{P}$ of $\sim 10^{9}-10^{10}$ years and low surface magnetic fields in the range $B \propto \sqrt{(P \dot{P})} \sim 10^{8}-10^{10} \mathrm{G}$.

At present, about $50 \%$ of all X-ray detected rotation-powered radio pulsars are MSPs (cf. Bogdanov et al. 2006, and references therein). Among them an extraordinarily rich astrophysics binary system which is formed by the millisecond pulsar PSR B1957+20 and its $0.025 M_{\odot}$ low mass white dwarf companion (Fruchter et al. 1988b). The binary period of the system is $9.16 \mathrm{~h}$. The spin period of the pulsar is $1.6 \mathrm{~ms}$ which is the third shortest among all known MSPs. Its period derivative of $\dot{P}=1.69 \times 10^{-20} \mathrm{~s} \mathrm{~s}^{-1}$ implies a spin-down energy of $\dot{E}=10^{35} \mathrm{erg} \mathrm{s}^{-1}$, a characteristic spin-down age of $>2 \times$ $10^{9}$ years, and a dipole surface magnetic field of $B_{\perp}=1.4 \times$ $10^{8}$ Gauss. Optical observations by Fruchter et al. (1988a) and van Paradijs et al. (1988) revealed that the pulsar wind consisting of electromagnetic radiation and high-energy particles is ablating and evaporating its white dwarf companion star. This rarely observed property gave the pulsar the name black widow pulsar. Interestingly, the radio emission from the pulsar is eclipsed for approximately $10 \%$ of each orbit by material expelled from the white dwarf companion. For a radio dispersion measure inferred distance of $1.5 \mathrm{kpc}$ (Taylor \& Cordes 1993) the pulsar moves through the sky with a supersonic velocity of $220 \mathrm{~km} \mathrm{~s}^{-1}$. The interaction of a relativistic wind flowing away from the pulsar with the interstellar medium (ISM) produces an $\mathrm{H}_{\alpha}$ bow shock which was the first one seen around a "recycled" pulsar (Kulkarni \& Hester 1988).

In 1992 Kulkarni et al. published a contour map of the X-ray emission of PSR B1957+20 which was derived from ROSAT PSPC observations. Although this ROSAT data were very sparse in statistics it led the authors to predict faint diffuse X-ray emission with constant surface brightness to be present along a cylindrical trail formed when the relativistic pulsar wind expands into pressure equilibrium with the interstellar medium behind the nebula. The much improved sensitivity of the Chandra and XMM-Newton observatories made it possible to probe and investigate the structure and properties of this unique binary system in much higher detail than it was possible with ROSAT, ASCA or BeppoSAX. A narrow X-ray tail with the extent of 16 arcsec and the orientation to the north-east was detected from it in deep Chandra observations by Stappers et al. (2003).

Searching in ROSAT data for a modulation of the pulsar's X-ray emission as a function of its orbital phase revealed a suggestive but insignificant increases in flux before (at phase $\phi \sim$ 0.17 ) and after (at phase $\phi \sim 0.4-0.5$ ) the pulsar radio eclipse $(\phi=0.25)$ (Kulkarni et al. 1992). Taking Chandra data into account revealed a hint that the lowest and highest fluxes are located during and immediately after the radio eclipse, respectively. The statistical significance of this modulation observed by Chandra, though, is only $98 \%$ and thus prevents any final conclusion on it (Stappers et al. 2003).

In this paper we report on XMM-Newton observations of PSR B1957+20 and its white dwarf companion. The paper is organized as follows. In Sect. 2 we describe the observations and data analysis. Section 3 gives a summary and discussion.

\section{Observations and data analysis}

PSR B1957+20 was observed with XMM-Newton on October 31, 2004 for a $30 \mathrm{ks}$ effective exposure. In this observation, the EPIC-MOS1 and MOS2 instruments were operated in full-frame mode using the thin filter to block 


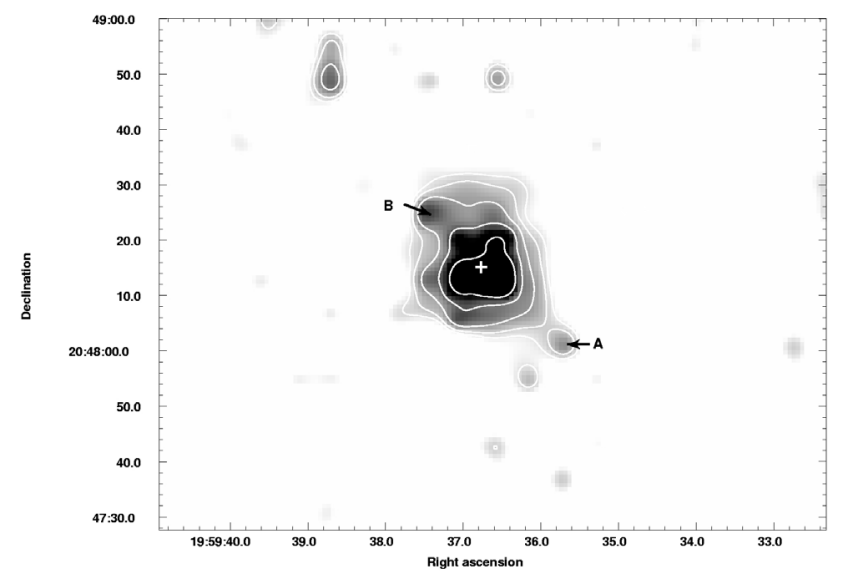

Fig. 1. XMM-Newton MOS1/2 image of the PSR B1957+20 -WD system with contour lines overlaid. The contour lines are at the levels of $(5.1,6.1,9.4,16.3,32.4,69.0) \times 10^{-6} \mathrm{cts} \mathrm{s}^{-1} \operatorname{arcsec}^{-2}$. The position of the pulsar is indicated.

optical stray light. The EPIC-PN detector was setup to operate in the fast timing mode. Because of the reduced spatial information provided by the $\mathrm{PN}$ in timing mode we use the MOS1/2 data for imaging and spectral analysis of the pulsar and its diffuse X-ray nebula while the PN data having a temporal resolution of $0.02956 \mathrm{~ms}$ allowed us to search for X-ray pulsations from the pulsar. All the data were processed with the XMM-Newton Science Analysis Software (SAS) package (Version 6.5.0). Spatial and spectral analyses were restricted to the $0.3-10.0 \mathrm{keV}$ energy band while for timing analysis events were selected for the energy range $0.3-3.0 \mathrm{keV}$.

\subsection{Spatial analysis}

Figure 1 shows the combined EPIC-MOS1/2 image of the PSR B1957+20 system. The image was created with a binning factor of 6 arcsec and by using an adaptive smoothing algorithm with a Gaussian kernel of $\sigma<4$ pixels in order to better make visible faint diffuse emission. The extent of the diffuse emission with its orientation to the north-east is about 16 arcsec which is consistent with the previous result derived from the Chandra Observations. However, the detailed structure of the X-ray emission from XMM-Newton can not be as clearly seen as from Chandra due to the 10 times wider Point Spread Function (PSF) of XMM-Newton.

Inspecting the XMM-Newton MOS1/2 image two faint features (denoted as A and B) which contribute to only about $3 \%$ of the total X-ray flux are apparent. In order to investigate whether this faint features are associated with nearby stars we inspected the Digitized Sky Survey data (DSS) and the USNO-B1.0 Catalogue for possible sources. These catalogues which are limited down to 22 mag (Krongold et al. 2001) and 21 mag (Monet et al. 2003) do not reveal possible counterparts. These features are not seen in the Chandra image though (Stappers et al. 2003).

\subsection{Spectral analysis}

Combined EPIC-MOS1/2 data of PSR B1957+20 were extracted from a circle of 30 arcsec radius centered at the pulsar position $\left(\mathrm{RA}(\mathrm{J} 2000)=19^{\mathrm{h}} 59^{\mathrm{m}} 36^{\mathrm{s}} \mathrm{s} 7\right.$, Dec $\left.=20^{\circ} 48^{\prime} 15^{\prime \prime} .12\right)$. The selection region contains about $85 \%$ of all source counts. Background photons were selected from a source-free region

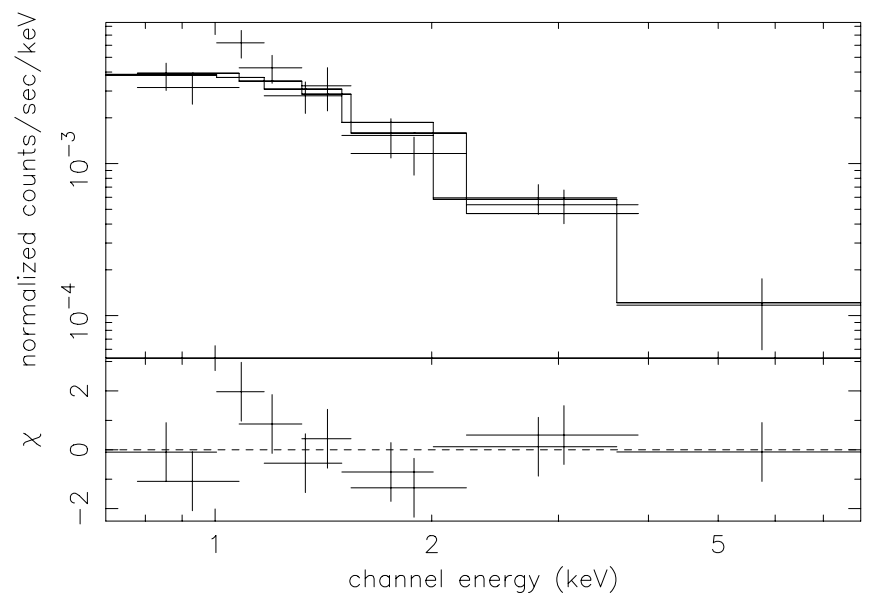

Fig. 2. Energy spectrum of PSR B1957+20 obtained from the XMM-Newton MOS1/2 data. The plot shows the X-ray spectrum fitted with an absorbed power law model (upper panel) and contribution to the $\chi^{2}$ fit statistic (lower panel).

near to the pulsar position. Response files were derived by using the XMM-Newton SAS tasks RMFGEN and ARFGEN.

After subtracting background photons, in total 338 sources counts were available for a spectral analysis. The extracted spectra were binned with at least 30 source counts per bin. Assuming that the emission originates from the interaction of the pulsar wind with the ISM or with the stellar wind we expect synchrotron radiation to be the emission mechanism of the detected X-rays. To test this hypothesis we fitted the spectrum with a power law model. Indeed, this model describes the observed spectrum with a reduced $\chi_{v}^{2}$ of 1.09 (for 8 d.o.f.). The photonindex is found to be $\alpha=2.03_{-0.36}^{+0.51}$. The column absorption $N_{\mathrm{H}}$ is $8.0 \times 10^{20} \mathrm{~cm}^{-2}$. For the normalization at $1 \mathrm{keV}$ we find $1.5_{-0.3}^{+0.9} \times$ $10^{-5}$ photons $\mathrm{keV}^{-1} \mathrm{~cm}^{-2} \mathrm{~s}^{-1}$ (1- $\sigma$ confidence for 1 parameter of interest). The spectrum (data and model) and the fit residuals are shown in Fig. 2.

The unabsorbed X-ray fluxed derived from the best fitting model parameters is $f_{\mathrm{x}}=8.35 \times 10^{-14} \mathrm{erg} \mathrm{s}^{-1} \mathrm{~cm}^{-2}$ and $f_{\mathrm{x}}=$ $7.87 \times 10^{-14} \mathrm{erg} \mathrm{s}^{-1} \mathrm{~cm}^{-2}$ in the $0.3-10 \mathrm{keV}$ and $0.1-2.4 \mathrm{keV}$ energy band, respectively. The X-ray luminosities in these energy bands - calculated for a pulsar distance of $1.5 \mathrm{kpc}-$ are $L_{\mathrm{x}}(0.3-10.0 \mathrm{keV})=2.24 \times 10^{31} \mathrm{erg} \mathrm{s}^{-1}$ and $L_{\mathrm{x}}(0.1-2.4 \mathrm{keV})=$ $2.12 \times 10^{31} \mathrm{erg} \mathrm{s}^{-1}$, respectively. The conversion efficiency $L_{\mathrm{x}} / \dot{E}$ in the $0.1-2.4 \mathrm{keV}$ band is found to be $\sim 2.12 \times 10^{-4}$.

In order to check whether the spectral emission characteristics changes for photons detected in a smaller compact region of 10 arcsec radius at the pulsar position we applied a spectral fit to this events only. The encircled energy within 10 arcsec is $60 \%$. In total, 186 sources counts were available for spectral fits. We did not find any significant change in the spectral parameters than reported above.

\subsection{Timing analysis}

The EPIC-PN camera observed the pulsar in the fast timing mode. In this mode the spatial and spectral information from a $64 \times 199$ CCD pixel array is condensed into a one dimensional $64 \times 1$ pixel array (1D-image), i.e. the spatial information in $Y$-direction is lost due to the continuous read-out of the CCD. The complete photon flux (source plus DC emission from foreground or background sources located along the read-out 
Table 1. List of the first and the last photon arrival times and its corresponding orbital phase of PSR B $1957+20$ for the MOS1/2 and PN detectors. Arrival times are corrected to the solar system barycentre. The orbital phase is measured from the time of ascending node.

\begin{tabular}{cccccc}
\hline \hline \multirow{2}{*}{ Date set } & \multicolumn{2}{c}{ 1st photon } & \multicolumn{2}{c}{ last photon } & Duration (s) \\
\cline { 2 - 5 } & Time (MJD) & Orbital phase & Time (MJD) & Orbital phase & \\
\hline MOS1 & 53309.9688 & 0.3605 & 53310.2830 & 0.1830 & 27143.4 \\
MOS2 & 53309.9666 & 0.3548 & 53310.2846 & 0.1871 & 27467.1 \\
PN & 53309.9791 & 0.3873 & 53310.3301 & 0.3063 & 30327.4 \\
\hline
\end{tabular}

direction) is accumulated and collapsed in the final 1D-image, severely reducing the signal-to-noise ratio of pulsed emission and preventing the detection of weak X-ray pulsations from the target of interest.

In order to search for X-ray pulsations from PSR B1957+20 we extracted 1639 counts from the CCD columns 33-41 in which the pulsar got located. In order to increase the signalto-noise ratio we restricted the analysis to the energy range $0.3-3.0 \mathrm{keV}$. Below and beyond this energy band the accumulative sky and instrument background noise exceeds the contribution from the pulsar itself (cf. Becker \& Aschenbach 2002). However, still $80 \%$ of the counts are estimated to be derived from the background.

The photon arrival times were corrected to the solar system barycentre with the BARYCEN tool (version: 1.17.3, JPL DE200 Earth ephemeris) of the SAS package. As the pulsar is in a binary we corrected for the orbital motion of the pulsar by using the method of Blandford \& Teukolsky (1976).

As millisecond pulsars are known to be extremely stable clocks we used the pulsar ephemeris from the ATNF Catalogue, $f=622.122030511927 \mathrm{~Hz}$ and $\dot{f}=-6.5221 \times 10^{-15} \mathrm{~s}^{-2}$ (at MJD $=48196.0$ ) to perform a period folding. By using the $Z_{n}^{2}$ statistics (Buccheri et al. 1983) with the harmonics number $(n)$ from one to ten no significant signal was detected at the radio spin period extrapolated for the epoch of the XMM-Newton observation. Restricting the period search to the various smaller energy bands did not change the result. A pulsed fraction upper limit of $9 \%(1-\sigma)$ is deduced by assuming a sinusoidal pulsed profile.

Arons \& Tavani (1993) predicted that depending on the flow speed and the degree of absorption and/or scattering by the companion wind the X-ray emission from PSR B1957+20 increases by up to a factor of 2.2 at the orbital phase before and after the radio eclipse. In order to test this prediction we created a lightcurve by binning all events in bins of $1.5 \mathrm{ks}$ width. With an effective exposure time of about $30 \mathrm{ks}$ and an orbit period of 9.16-h the XMM-Newton MOS1/2 and PN data cover roughly $83 \%$ and $92 \%$ of one binary orbit, respectively. Table 1 lists the first and the last photon arrival times recorded by the MOS1/2 and EPIC-PN detectors and the corresponding pulsar binary orbital phase. The lightcurves resulting from the EPIC-PN and MOS1/2 data are shown in Fig. 3. For the lightcurve deduced from the PN-data (upper solid curve) it is clearly seen that before the radio eclipse, i.e. between the orbital phase 0.1 and 0.25 , the X-ray emission increases. The emission in the highest bin is about a factor of 3.0 stronger than at other orbital phase angles before and after the radio eclipse. This is in agreement with the predictions made by Arons \& Tavani (1993).

Although it is the first time that a significant X-ray flux modulation from PSR B1957+20 is observed, the flux increase near the phase of the radio eclipse is virtually only seen in the PN data. Owing to the short observation time of $30 \mathrm{ks}$, which is less than the time of one full binary orbit, the orbit angles $0.30-0.38$ and $0.18-0.36$ are not covered by EPIC-PN

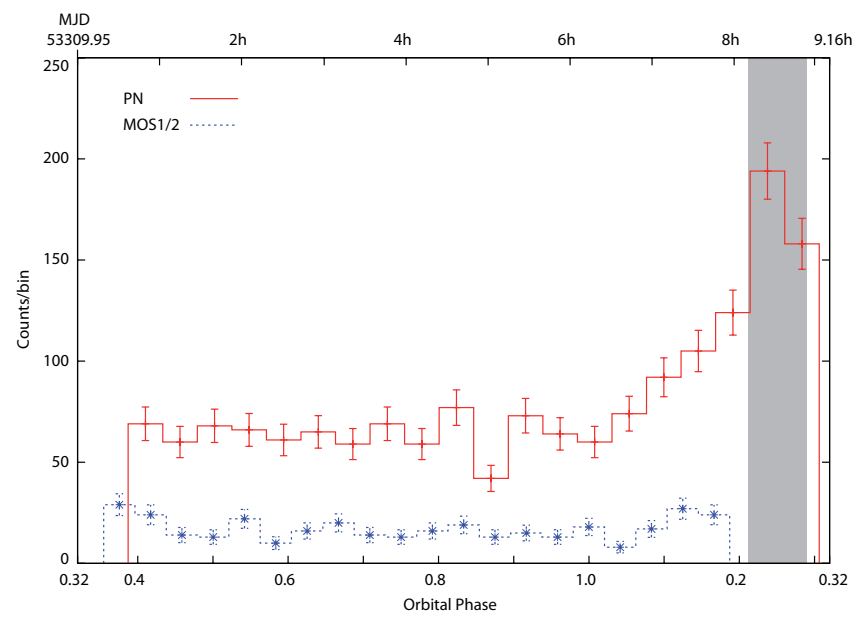

Fig. 3. X-ray emission from PSR B $1957+20$ within $0.3-3.0 \mathrm{keV}$ as function of the pulsar's orbital phase $(\phi)$. We mapped one complete orbital period of this system at the starting point of MJD $=53309.95$, i.e. $\phi=0.32 . \phi=1.0$ corresponds to the ascending node of the pulsar orbit. The upper curve was obtained from the XMM-Newton EPIC-PN (background level at $66 \mathrm{cts} / \mathrm{bin}$ ). The lower lightcurve is obtained from the MOS1/2 data. The gray strip between the orbital angle 0.21-0.29 indicates the eclipse of the pulsar. Phase bins with zero counts correspond to phase angles not covered in the observation.

and MOS1/2 data at all. It thus turns out that it was a great fortune that the EPIC-PN, more or less by chance, covered the orbital phase range of the radio eclipse and provided us evidence for the flux enhancement while the MOS1/2 detectors were already switched off.

\section{Summary and discussion}

Interaction between relativistic pulsar winds which carry away the rotational energy of pulsars and the surrounding medium is expected to create detectable X-ray emission. Indeed, there are about 30 pulsar wind nebulae (PWNe) currently detected in the X-ray band (e.g. Kaspi et al. 2006; Gaensler \& Slane 2006; Kargaltsev \& Pavlov 2006). However, these PWNe are all powered by young and powerful pulsars with spin-down energies of more than $\sim 3.6 \times 10^{36} \mathrm{erg} \mathrm{s}^{-1}$. Until now, only two MSPs are known to have X-ray nebulae. They are PSR B1957+20 (Stappers et al. 2003) and PSR J2124-3358 (Hui \& Becker 2006). Both of them have tail-like structures behind the moving pulsars. These trails could be associated with the shocked relativistic wind confined by the ram pressure of the ambient ISM.

The XMM-Newton data of PSR B1957+20 have provided observational evidence for a strong dependence of the pulsar's $\mathrm{X}$-ray emission on its binary orbital phase. It is the first time that a significant X-ray flux modulation from PSR B1957+20 is observed. The emission near to the radio eclipse is supposed to be beamed in a forward cone because the shocked fluid is accelerated by the pressure gradient as it flows around the 
eclipse region. Relativistic beaming would tend to give the maximum flux just before and after the pulsar eclipse which for PSR B 1957 +20 is at orbital phase $=0.25$. Arons $\&$ Tavani (1993) predicted that the immediately downstream flow velocity of the shocked pulsar wind from the shock area along the line between the pulsar and the ablating companion is about $c / 3$ and is even higher behind the relativistic shock when it passes around the companion. The tenuous relativistic plasma accelerates as it flows around the companion, possibly passing through a sonic transition to leave the binary system with the velocity larger than $c / \sqrt{3}$. Due to Doppler boosting, the probable post-shock velocities of the relativistic wind then suggest X-ray emission variation around the orbital phase by a factor between $1.3(v=c / 3)$ and $2.2(v \cong c / \sqrt{3})$. This numbers were estimated without considering the effect of absorption and/or scattering within the binary. On the contrary, the X-ray emission at the eclipse may be reduced because of the obscuration of the shock by the companion.

However, given the limited photon statistics of the XMM-Newton data it is not possible to investigate any binaryphase resolved imaging and spectral variation as a function of orbit phase or to determine the exact geometry of the peak emission. Such analysis would allow us to investigate with higher accuracy than currently possible whether the X-ray emission from PSR B1957+20 is present during all orbit angles or virtually only near to the radio eclipse while diffuse X-ray emission from the PWN is present at any orbital angle. Indeed, the later is suggested by the current data and a confirmation would have a severe impact on our understanding of the pulsar's X-ray emission properties.

As the present XMM observation covers barely one binary orbit, we stress that it can not be fully excluded that the increase in photon flux near the orbital angle of the radio eclipse is due to a single "burst like" event or that the peak flux emission varies from orbit to orbit or on longer time scales. Clearly, a repeated coverage of the binary orbit in a longer XMM-Newton observation and a comparison with the 2004 data would answer this question immediately. This, in addition, would provide us not only a better photon statistic but would also allow us to determine the emission geometry with a much higher accuracy than currently possible.

Acknowledgements. This work made use of the XMM-Newton data archive. The first author acknowledges the recipe of funding provided by the Max-Planck Society in the frame of the International Max-Planck Research School (IMPRS).

\section{References}

Alpar, M. A., Cheng, A. F., Ruderman, M. A., \& Shaham, J. 1982, Nature, 300, 728

Arons, J., \& Tavani, M. 1993, ApJ, 403, 249

Becker, W., \& Aschenbach, B. 2002, nsps. conf, 64

Blandford, R., \& Teukolsky, S. A. 1976, ApJ, 205, 580

Buccheri, R., Bennett, K., Bignami, G. F., et al. 1983, A\&A, 128, 245

Bogdanov, S., Grindlay, J. E., Heinke, C. O., et al. 2006, ApJ, 646, 1104

Fruchter, A. S., Gunn, J. E., Lauer, T. R., \& Dressler, A. 1988a, Nature, 334, 686

Fruchter, A. S., Stinebring, D. R., \& Taylor, J. H. 1988b, Nature, 333, 237

Gaensler, B. M., \& Slane, P. O. 2006, ARA\&A, 44, 17

Hui, C. Y., \& Becker, W. 2006, A\&A, 448, L13

Kargaltsev, O. Y., \& Pavlov, G. G. 2006, in preparation

Kaspi, V. M., Roberts, M. S. E., \& Harding, A. K. 2006, in Compact Stellar X-ray Sources, ed. W. H. G. Lewin, \& M. van der Klis (Cambridge University Press), 279

Krongold, Y., Dultzin-Hacyan, D., \& Marziani, P. 2001, AJ, 121, 702

Kulkarni, S. R., \& Hester, J. J. 1988, Nature, 335, 801

Kulkarni, S. R., Phinney, E. S., Evans, C. R., \& Hasinger, G. 1992, Nature, 359, 300

Manchester, R. N., Hobbs, G. B., Teoh, A., \& Hobbs, M. 2005, AJ, 129, 1993

Monet, D. G., Levine, S. E., Canzian, B., et al. 2003, AJ, 125, 984

Stappers, B. W., Gaensler, B. M., Kaspi, V. M., van der Klis, M., \& Lewin, W. H. G. 2003, Science, 299, 1372

Taylor, J. H., \& Cordes, J. M. 1993, ApJ, 411, 674

van Paradijs, J., Allington-Smith, J., Callanan, P., Hassall, B. J. M., \& Charles, P. A. 1988 , Nature, 334,684 